\title{
Basisstandards einer Fachabteilung für Herzchirurgie
}

\section{Qualitätsanforderungen für die Versorgung von herzkranken Patientinnen und Patienten im Zusammenhang mit Operationen/Eingriffen am Herzen, den herznahen Gefäßen und der thorakalen Organtransplantation}

\author{
Andreas Beckmann $^{1} \quad$ F. Beyersdorf ${ }^{2} \quad$ A. Diegeler ${ }^{3} \quad$ F. W. Mohr ${ }^{4} \quad$ A. Welz ${ }^{5} \quad$ J.-G. Rein ${ }^{1} \quad$ J. Cremer $^{6}$ \\ ${ }^{1}$ Deutsche Gesellschaft für Thorax-, Herz- und Gefäßchirurgie \\ [DGTHG], Berlin, Germany \\ 2 Klinik für Herz- und Gefäßchirurgie, Universitäts-Herzzentrum \\ Freiburg-Bad Krozingen, Germany \\ ${ }^{3}$ Klinik für Kardiochirurgie, Herz- und Gefäßklinik GmbH Bad, \\ Neustadt, Germany \\ ${ }^{4}$ Klinik für Herzchirurgie, Herzzentrum Leipzig GmbH, Leipzig, \\ Germany \\ ${ }^{5}$ Klinik und Poliklinik für Herzchirurgie, Rheinische F.-W.-Universität, \\ Bonn, Germany \\ ${ }^{6}$ Klinik für Herz- und Gefäßchirurgie, University Hospital Schleswig- \\ Holstein, Campus Kiel, Germany \\ Thorac Cardiovasc Surg 2013;61:651-655.
}
Abstract
Keywords
- outcomes
- quality of life
- health economics
- quality assurance
- cardiac surgery
- patient care

Current evolutions and substantial amendments of the German health care system in combination with distinguished progress in cardiac surgery over the past years require both a reflection of principles in patient-centered care and an update of basic standard requirements for a department of cardiac surgery in Germany. In due consideration of the data from the voluntary registry of the German Society for Thoracic and Cardiovascular Surgery, this article accurately defines core requirements for a cardiac surgical department (cardiac surgery on-site), subdivided into facilities, staff and processes. If based on these standards, one may anticipate that cardiac surgical care is performed under appropriate conditions leading to an intrinsic benefit for patients.

\section{Präambel}

Bereits in den Jahren $1976^{1}$ bzw. $1993^{2}$ haben sich eigens eingesetzte Kommissionen der Deutschen Gesellschaft für Thorax-, Herz- und Gefäßchirurgie (DGTHG) mit verschiedenen Fragen zu „Voraussetzungen zum Betrieb leistungsfähiger Kliniken“ bzw. zu „Qualitätsstandards in der Herzchirurgie“ eingehend befasst. Die zwischenzeitlichen Entwicklungen und umfassenden Veränderungen im deutschen Gesundheitswesen - wie beispielsweise mehrere Krankenhausreformen, die Einführung eines pauschalierenden Vergütungssystems

received

September 5, 2013

accepted

September 6, 2013

published online

September 26, 2013
(G-DRG), die kontinuierliche Etablierung facettenreicher Elemente des Qualitätsmanagements, die Zentrumsbildung und nicht zuletzt auch die Entwicklung des Fachgebiets Herzchirurgie selbst - machen es erforderlich, sich mit den Grundlagen der Patientenversorgung im Bereich der Herzmedizin erneut zu befassen und novellierte Basisstandards einer Fachabteilung für Herzchirurgie konkret zu definieren.

Als Datengrundlage in diesem Zusammenhang dient die bereits seit Jahrzehnten etablierte DGTHG-Leistungsstatistik $^{\mathcal{O}}$, die eine jährlich aktualisierte Übersicht zu allen herzchirurgischen Leistungen in Deutschland liefert. Diese zeigt
DOI http://dx.doi.org/ 10.1055/s-0033-1357260. ISSN 0171-6425. (c) 2013 Georg Thieme Verlag KC Stuttgart · New York 
für das Jahr 2012, dass in 80 Fachabteilungen etwa 125.000 Operationen am Herzen und den herznahen Gefäßen durchgeführt wurden. Hierbei handelte es sich um 55.302 koronarchirurgische Operationen, 28.521 Herzklappen-Eingriffe, 25.232 Herzschrittmacher- oder AICD-Operationen, 7.380 Aorten-Eingriffe, 5.593 Operationen bei angeborenen Herzfehlern, 2618 Operationen von Herzunterstützungssystemen, 2.473 andere herzchirurgische Eingriffe, 327 Herz-, 315Lungen und 15 Herz-Lungen-Transplantationen. ${ }^{3}$

Im Hinblick auf die Qualität der herzchirurgischen Versorgung in Deutschland ist festzustellen, dass diese - insbesondere auch im internationalen Vergleich - auf einem kontinuierlich hohen Niveau erfolgt. Dies zeigt sich u.a. durch bemerkenswert hohe Überlebensraten der Patienten nach herzchirurgischen Operationen trotz Anstieg der präoperativen Risikoprofile. Letztere sind eine unmittelbare Folge des demographischen Wandels mit einer ständigen Zunahme von Patienten höheren Lebensalters und den damit einhergehenden Komorbiditäten. Ferner ist die Gesamtzahl der Herzoperationen in den letzten 10 Jahren weitgehend konstant und die Anzahl der Fachabteilungen für Herzchirurgie nahezu unverändert. Über denselben Zeitraum haben sich die Wartezeiten für Patienten, die einen herzchirurgischen Eingriff benötigen, auf weniger als 4 Wochen reduziert, so dass von einer flächendeckenden und angemessenen herzchirurgischen Versorgung in Deutschland auszugehen ist.

Diese Fakten sowie auch die Tatsachen, dass in den vergangenen Jahren perioperative Qualitätsstandards etabliert, bewährte bzw. neue Operationstechniken entwickelt, Grundlagen erforscht und Innovationen in der Herzchirurgie realisiert wurden, erfordern eine Novellierung bisheriger Standardvorgaben mit dem Ziel, die Qualität herzchirurgischer Fachabteilungen in Deutschland durch eine Definition obligater Qualitätsmerkmale auch weiterhin sicherzustellen.

Daher beschreibt die vorliegende Publikation im Sinne von „Basisstandards“ Grundlegendes - unterteilt nach Infrastrukturen, Personal und Prozessen - für eine angemessene Versorgung von Patientinnen und Patienten mit Herzkrankheiten, bei denen Operationen/Eingriffe am Herzen, den herznahen Gefäßen oder thorakale Organtransplantationen durchgeführt werden.

\section{Infrastrukturelle Voraussetzungen}

Unter Berücksichtigung der langjährigen Realitäten und bisherigen Erfahrungen erscheint es zielführend, ein Krankenhaus mit einem geeigneten Organisationsmodell differenziert zu gliedern um die komplexen Anforderungen der modernen Krankenversorgung erfüllen zu können. Als Grundlage für dieses spezielle Konstrukt wurde im Jahr 2003 eine Deutsche Industrienorm (DIN) veröffentlicht, die konkrete Empfehlungen gibt und als DIN 13080 die „Gliederung des Krankenhauses in Funktionsbereiche und Funktionsstellen “4 vorsieht.

Betrachtet man im Weiteren nun die Funktionsbereiche näher, gelangt man zwangsläufig zu dem Begriff „Fachabteilung“, der in verschiedenen Kontexten und Erhebungen verbindlich genutzt wird.
Beispielsweise ist gemäß den Grunddaten der deutschen Krankenhausstatistik ${ }^{5}$ ein Krankenhaus in Einheiten organisiert, die unter anderem aus konkret ausgewiesenen Fachabteilungen bestehen. Der Begriff Fachabteilung (FA) wird als eine „organisatorisch abgegrenzte, von Ärztinnen/Ärzten ständig verantwortlich geleitete Abteilung mit für den jeweiligen Fachbereich besonderen Behandlungseinrichtungen“ definiert. ${ }^{5}$ Hierbei orientiert sich die Fachabteilungsgliederung (bis auf wenige Ausnahmen) seit dem Jahr 2002 an den fachärztlichen Gebiets- bzw. Schwerpunktbezeichnungen der Musterweiterbildungsordnung der Bundesärztekammer. Seit diesem Zeitpunkt wird die im Folgenden näher zu betrachtende Fachabteilung für Herzchirurgie in der Liste der Bezeichnungen explizit ausgewiesen. Ergänzend wird bei der Fachabteilungsgliederung darauf hingewiesen, dass es sich um Abteilungen handelt „die von einem fachlich nicht weisungsgebundenen Arzt mit entsprechender Fachgebietsbezeichnung geleitet werden und die für dieses Fachgebiet überwiegend genutzt werden. ${ }^{\text {}} 5$

\section{Ambulanz}

Um Patienten sektorenübergreifend vor und nach einer Herzoperation zur jeweiligen Herzerkrankung individuell beraten, adäquat untersuchen, umfassend informieren und über mögliche Therapieoptionen aufklären zu können, ist eine der Fachabteilung Herzchirurgie zugehörige Ambulanz mit angemessenen Räumlichkeiten und medizinisch-technischer Ausstattung erforderlich. Neben der perioperativ stationären Behandlung gehört zur herzchirurgischen Versorgung auch die mittel- und langfristige Fortführung der Betreuung und Therapie nach erfolgreicher Herzoperation, dies sowohl im Hinblick auf operationsassoziierte Besonderheiten und Folgen des operativen Zugangs, als auch insbesondere bezüglich der weiteren Genesungsverläufe oder aber der Notwendigkeit der kontinuierlichen Behandlung von Patienten mit chronischen Herzleiden.

\section{Periphere herzchirurgische Pflegestation}

Eine Fachabteilung für Herzchirurgie verfügt mindestens über eine periphere Pflegestation mit wenigstens 20 Betten um die Herzpatienten in allen Phasen des prä- und postoperativen Behandlungsverlaufs adäquat therapieren zu können. Mindestens 20\% der Betten der peripheren Pflegestation verfügen über ein bettseitiges Monitoring mit EKG-, Blutdruck- und $\mathrm{O}_{2}$-Sättigungs-Überwachung. Diese Erfordernisse ergeben sich aus der Tatsache, dass im Vergleich zu anderen Fachdisziplinen der Betreuungsaufwand herzkranker Menschen auch auf einer peripheren Pflegestation höher ist, weil es zumeist multimorbide Patienten sind, die häufig unter Einsatz der extrakorporalen Zirkulation operiert werden. Dies resultiert in einer perioperativ umfänglicheren Überwachungsintensität (Herz-Kreislauf-Monitoring) und Betreuungsbedürftigkeit durch qualifizierte Ärzte, geschultes Pflegepersonal und erfahrene Physiotherapeuten. Beispielhaft seien einige patientenassoziierte Risiken, die zumeist passager auftreten, genannt: brady- und tachykarde Herzrhythmusstörungen, 
psychopathologische Auffälligkeiten, akute Nachblutungen, Pleuraergüsse, Herzbeuteltamponaden oder akute Herzinsuffizienz.

\section{Herzchirurgische Intermediate Care Station (IMC)}

Eine Fachabteilung für Herzchirurgie sollte über eine herzchirurgische Intermediate Care Station/Einheit verfügen. Diesbezügliche Empfehlungen zur personellen, infrastrukturellen und apparativen Ausstattung einer herzchirurgischen IMC wurden von der DGTHG bereits im Jahre 2012 publiziert. $^{6}$ Als wesentliche Notwendigkeiten für die Einrichtung einer IMC werden die bereits präoperativ intensive Behandlungsbedürftigkeit einiger Patienten, die Reduktion der Aufenthalte auf der Intensivstation sowie die Minimierung potentieller Rückverlegungen auf die Intensivstation gesehen. Weitere Einzelheiten bzw. konkrete Vorgaben sind der entsprechenden Publikation zu entnehmen ${ }^{6}$ und bedürfen daher an dieser Stelle keiner weiteren Ausführungen.

\section{Herzchirurgische Intensivtherapiestation}

Eine Fachabteilung für Herzchirurgie muss über eine herzchirurgische oder interdisziplinäre Intensivtherapiestation verfügen. Die zuletzt im Jahr 2013 durchgeführte Umfrage zur Struktur herzchirurgischer Intensivstationen zeigt, dass die Fachabteilungen für Herzchirurgie auch weiterhin die chirurgische Disziplin mit den meisten Intensivstationsbetten sind. ${ }^{7}$ Hinsichtlich der vorzuhaltenden Struktur und Ausstattung hat die Deutsche Interdisziplinäre Vereinigung für Intensiv- und Notfallmedizin (DIVI) bereits im Jahr 2010 „Empfehlungen zur Struktur und Ausstattung von Intensivtherapiestationen“ publiziert, die auch als Minimalstandard für die Behandlung herzchirurgischer Intensivpatienten angesehen werden können. ${ }^{8}$ Ferner gilt es die in ihrer aktuellen Fassung bis zum 01.04.2015 gültige, interdisziplinär erstellte S3-Leitlinie "Intensivmedizinische Versorgung herzchirurgischer Patienten - Hämodynamisches Monitoring und Herz-Kreislauf-Therapie" ${ }^{\text {" }}$ zu beachten und ein den jeweiligen lokalen Gegebenheiten angepasstes, praxisorientiertes Konzept festzulegen und konsequent umzusetzen. Hinsichtlich der ärztlichen Versorgung ist eine permanente Arztpräsenz (24 / 7 / 365) zu gewährleisten.

\section{Operationseinheit}

Eine Fachabteilung für Herzchirurgie hält mindestens 2 Operationssäle einschließlich geeigneter Räumlichkeiten zur Durchführung der anästhesiologischen Vorbereitung und Nachbetreuung vor.

Die Operationssäle müssen eine dem technischen Fortschritt und wissenschaftlichen Kenntnisstand entsprechende Grundausstattung haben, jederzeit verfügbar sein und obligat die Voraussetzungen zum Einsatz von Herz-Lungen-Maschinen, extrakorporalen Herz-Kreislaufunterstützungs-Systemen (z.B. ECMO, ECLS, VAD), transösophagealen Echokardiographien (TEE), Nierenersatzverfahren sowie Röntgen-/Durchleuchtungsund Bronchoskopiegeräten erfüllen.
Hinsichtlich der hygienischen und infektionspräventiven Maßnahmen sind neben dem Infektions schutzgesetz (IfSG $)^{10}$ und dem Gesetz zur Änderung des Infektionsschutzgesetzes ${ }^{11}$ auch die entsprechenden Vorgaben für den Operationsbereich der Richtlinie für Krankenhaushygiene und Infektionsprävention des Robert-Koch-Instituts $^{12}$ umzusetzen.

Aktuelle Entwicklungen zeigen, dass in absehbarer Zeit von einer Verfügbarkeit sogenannter Hybrid-Operationssäle für alle herzchirurgischen Fachabteilungen ausgegangen werden kann. Diese sind eine wichtige infrastrukturelle Voraussetzung für die qualifizierte interdisziplinäre Durchführung bestimmter konventioneller Operationen und katheterbasierter Prozeduren bei Patientenkollektiven, die durch Kooperation von Herzchirurgen und Kardiologen im sogenannten „Herzteam“ behandelt werden. In diesem Kontext gilt es zudem festzustellen, dass die transthorakale/-ösophageale Echokardiographie, als ein hervorragendes organbezogenes Bildgebungsverfahren, durchgängig peri- und intraoperativ in allen Bereichen der Fachabteilung für Herzchirurgie zur Verfügung stehen muss.

\section{Bereich Kardiotechnik}

Diese Organisationseinheit muss in unmittelbarer Nähe zur Operationseinheit liegen und über geeignete Räume verfügen, in denen jederzeit die hygienisch und medizintechnisch einwandfreien Vor- und Nachbereitungen der Einsätze von Herz-Lungen-Maschinen (Lagerung, Aufrüstung, Entsorgung, Wartung etc.), Herz-Kreislauf-Unterstützungssystemen (z.B. IABP, ECMO / ECLS) und Organersatzgeräten (Hämofiltration /-dialyse, VAD) vorgenommen werden können. Zudem sind entsprechend ausgestattete Lagerräume mit angemessenen Kapazitäten vorzuhalten.

\section{Personal und Qualifikation}

Eine Fachabteilung für Herzchirurgie muss von einem Facharzt/Fachärztin für Herzchirurgie geleitet werden. Daneben müssen weitere Fachärzte/Fachärztinnen für Herzchirurgie, die in der intensivmedizinischen Behandlung der herzoperierten Patienten verantwortlich eingebunden sind, mit einer zeitlichen Arbeitsleistung von mindestens 4 Vollzeitäquivalenten (VZÄ) zur Verfügung stehen. Mindestens einer der Fachärzte/Fachärztinnen verfügt über die Zusatzbezeichnung Intensivmedizin. Ein fachärztlicher Rufbereitschaftsdienst muss durchgängig zur Verfügung stehen. Neben den obligaten Fachärzten/Fachärztinnen müssen zudem weitere Ärzte/Ärztinnen, die sich gegebenenfalls in der Facharztweiterbildung für Herzchirurgie befinden, mit einer Arbeitsleistung von mindestens 5 VZÄ angestellt sein. Durch die Ärzte/Ärztinnen muss durchgängig ein Bereitschaftsdienst gewährleistet werden. Sofern durch die Ärzte der Fachabteilung für Herzchirurgie weitere Versorgungsaufgaben wahrgenommen werden (Intensivmedizin, Chirurgie angeborener Herzfehler, ${ }^{13}$ Organtransplantation etc.), sind für diese Funktionen gesonderte VZÄ zwingend erforderlich und gegebenenfalls gesonderte Bereitschaftsdienstregelungen zu treffen. 
Der Pflegedienst der Krankenstationen muss aus Gesundheits- und Krankenpflegerinnen oder -pflegern bestehen und in Mehrschichtdiensten organisiert sein. Bezüglich der Intensivtherapiestation müssen mindestens 25\% der Mitarbeiterinnen und Mitarbeiter des Pflegedienstes eine Fachweiterbildung zum Fachgesundheits- und Krankenpfleger/in für Intensivpflege und Anästhesie bzw. Fachgesundheitsund Kinderkrankenpfleger/in für Intensivpflege und Anästhesie, absolviert haben. In mindestens einer Schicht pro Tag muss auf der Intensivtherapiestation eine Pflegekraft mit abgeschlossener Fachweiterbildung anwesend sein. Der Pflegedienst der Operationseinheit muss von Gesundheitsund Krankenpflegerinnen oder -pflegern besetzt sein, die über mehrjährige Erfahrungen im Bereich der Herzchirurgie verfügen. Mindestens 20\% dieser erfahrenen Mitarbeiterinnen und Mitarbeiter müssen eine Weiterbildung zur Fachgesundheits- und Krankenpfleger/in im Operationsdienst abgeschlossen haben. Für die Fachabteilung für Herzchirurgie muss der Pflegedienst der Operationseinheit durchgängig einen Bereitschaftsdienst zur Verfügung stellen.

Das Anästhesiepflegepersonal muss von Gesundheits- und Krankenpflegerinnen oder -pflegern besetzt sein, die über Erfahrungen im Bereich der Herzchirurgie verfügen. Mindestens 20\% dieser erfahrenen Mitarbeiterinnen und Mitarbeiter müssen eine Weiterbildung zur Fachgesundheits- und Krankenpfleger/in für Intensivpflege und Anästhesie bzw. Fachgesundheits- und Kinderkrankenpfleger/in für Intensivpflege und Anästhesie, abgeschlossen haben. Für die Fachabteilung für Herzchirurgie muss das Anästhesiepflegepersonal durchgängig einen Bereitschaftsdienst zur Verfügung stellen.

Der Bereich Kardiotechnik verfügt über „Kardiotechniker/innen" mit nachweislicher Qualifikation (z.B. European Certificate in Cardiovascular Perfusion - ECCP; www.ebcp. org) mit einer Arbeitsleistung von mindestens 4 VZÄ. Beim Einsatz der extrakorporalen Zirkulation (EKZ) sind prinzipiell zwei Kardiotechniker und -innen erforderlich. Ein Rufbereitschaftsdienst von Kardiotechnikern/-innen muss durchgängig zur Verfügung stehen.

\section{Prozesse}

Die Mitarbeiter/-innen der Fachabteilung für Herzchirurgie sind eingebunden in ein interdisziplinäres, multiprofessionelles Team, das die qualitativ hochwertige Patientenversorgung unter Beachtung relevanter medizinischer Leitlinien in enger Kooperation gewährleistet.

Im Kontext einer qualitativ angemessenen Patientenversorgung erscheint es geboten, multiprofessionelle Herzteams obligat zu etablieren um konsentierte Therapieentscheidungen im Interesse der Patienten zu treffen.

Daher sind neben den bereits bei den personellen Anforderungen genannten Personen folgende qualifizierte Personengruppen in diverse Prozesse einzubeziehen:

- Fachärztinnen / Fachärzte für Kardiologie und Innere Medizin

- Fachärztinnen / Fachärzte für Anästhesiologie mit kardiochirurgischer Expertise
- Physiotherapeutinnen / Physiotherapeuten mit mehrjähriger Erfahrung in der Versorgung herzkranker Patientinnen und Patienten

- Hygienefachkraft mit einem dem Versorgungsumfang angemessenen VZÄ

Ferner müssen folgende Fachbereiche / Dienstleistungen im Sinne von Konsiliardiensten bzw. zur potentiellen Mitbehandlung der Patienten zur Verfügung stehen:

- Kardiologie

- Anästhesiologie

- Labormedizin inkl. Blutbank

- Radiologie

- Neurologie

- Gastroenterologie

- Angiologie / Gefäßchirurgie

- Thoraxchirurgie

- Allgemein- / Viszeralchirurgie

- Neurochirurgie

Im Hinblick auf das Qualitätsmanagement einschließlich der Qualitätssicherung hat eine Fachabteilung für Herzchirurgie vielfältige Aufgaben strukturiert zu erfüllen und muss unter anderem die umfänglichen, für das Fachgebiet gesetzlich verpflichtenden Dokumentationen ( $\$ 137$ SGB V) vornehmen, wofür qualifiziertes Personal zwingend notwendig ist.

\section{Zusammenfassung}

Eine qualitativ hochwertige Versorgung herzchirurgischer Patienten kann nur mit Einsatz qualifizierten Personals sowie unter der Voraussetzung geeigneter Infrastrukturen mit differenziert strukturierten Prozessen dauerhaft erfolgreich sein. Daher legt die vorliegende Publikation Basisstandards fest, deren Erfüllung zwar keine Garantie, jedoch eine nachvollziehbare Grundlage für die qualitativ angemessene Versorgung herzchirurgischer Patienten und deren Sicherheit darstellt. An dieser Stelle sei nochmals explizit angemerkt, dass die dargelegten Vorgaben als Basisvoraussetzungen zu verstehen sind, und dass für spezielle Leistungen des Fachbereichs Herzchirurgie (z.B. Organtransplantation, Chirurgie angeborener Herzfehler etc.) in allen Aspekten (Infrastruktur, Personal und Prozesse) zusätzliche Voraussetzungen obligat zu erfüllen sind.

\section{Danksagung}

Die Autoren (Engerer Vorstand der DGTHG) bedanken sich bei den Beisitzern des Vorstandes für die aktive Mithilfe bei der Erstellung dieses Manuskriptes: A. Böning, H. Niehaus, H. Reichenspurner, Ch. Schlensak, G. Trummer, Th. Walther.

\section{Literatur}

1 Borst HG, et al Bericht der Kommission für Kapazitätsermittlung der Deutschen Gesellschaft für Thorax-, Herz- und Gefäßchirurgie. Teil II: Voraussetzungen zum Betrieb leistungsfähiger Kliniken. Thorac Cardiovasc Surg 1976;24:261-271 
2 Leitz K-H, et al. Bericht der Kommission für Qualitätsstandards der Deutschen Gesellschaft für Thorax-, Herz- und Gefäßchirurgie: Qualitätsstandards in der Herzchirurgie. Thorac Cardiovasc Surg 1993;41:VII-XIV

3 Beckmann A, et al. Cardiac surgery in Germany during 2012: A report on behalf of the German Society for Thoracic and Cardiovascular Surgery. Thorac Cardiovasc Surg 2013:61;in press

4 DIN 13080: Gliederung des Krankenhauses in Funktionsbereiche und Funktionsstellen; Ausgabe 2033-07; NA 053-02-01 AABegriffe und Koordination im Krankenhaus

5 Krankenhausstatistik: Grunddaten Erhebung der Krankenhausstatistik-Verordnung (KHStatV) (BGBl. S. 2.135), Geltung in Verbindung mit dem Bundesstatistikgesetz (BStatG)

6 Markewitz A, et al. Empfehlungen der Deutschen Gesellschaft für Thorax-, Herz- und Gefäßchirurgie zur personellen, infrastrukturellen und apparativen Ausstattung einer herzchirurgischen Intermediate Care Station. ZHTG 2012;26:48-55

7 Markewitz A, et al. Status of cardiac surgical intensive care medicine in Germany in 2013: A report on behalf of the German
Society for Thoracic and Cardiovascular Surgery. Thorac Cardiovasc Surg 2013; in press

8 Jorch G, et al. Empfehlungen zur Struktur und Ausstattung von Intensivtherapiestationen. DIVI 2011;2(2):78-86

9 Carl Met al : S3-Leitlinie zur intensivmedizinischen Versorgung herzchirurgischer Patienten Hämodynamisches Monitoring und Herz-Kreislauf; AWMF Register 001/016; Publikation 01.04 .2010

10 "Infektionsschutzgesetz vom 20. Juli 2000 (BGBl. I S. 1045), das durch Artikel 5 Absatz 2 des Gesetzes vom 20. April 2013 (BGBI. IS. 868) geändert worden ist"

11 Gesetz zur Änderung des Infektionsschutzgesetzes und anderer Gesetze; BGBL 2011 Teil 1, Nr. 41; 03.Aug. 2011

12 Vorwort und Einleitung der Kommission zur Richtlinie für Krankenhaushygiene und Infektionsprävention; Bundesgesundheitsbl - Gesundheitsforsch - Gesundheitsschutz 2004; 47:409-411

13 Richtlinie Kinderherzchirurgie. Bundesanzeiger ISSN 0720-6100 G 1990; Jg 62; 18. Juni 2010; Nr 89a 